\title{
CARACTERIZAÇÃO SEDIMENTAR SUBSUPERFICIAL RECENTE DA PLANÍCIE COSTEIRA AMAZÔNICA: O EXEMPLO DA REGIÃO DO CABO NORTE - AP.
}

\author{
XAVIER, D.A. ${ }^{1 *}$; BARCELLOS, R.L. ${ }^{1}$ B BERRÊDO, J.F. ${ }^{2}$ \& SILVEIRA, O.M. ${ }^{3}$ \\ 1.Programa de Pós-Graduação em Oceanografia, Departamento de Oceanografia, Centro de Tecnolo- \\ gia e Geociências, Universidade Federal de Pernambuco, PE, Brasil. \\ 2.Programa de Estudo Costeiros, Centro de Ciências da Terra e Ecologia, Museu Paraense Emílio \\ Goeldi, PA, Brasil \\ 3.Laboratório Institucional de Oceanografia Geológica, Faculdade de Oceanografia, Universidade \\ Federal do Pará Instituto de Geociências, PA, Brasil. \\ *Corresponding author: diego.a.xavier@gmail.com
}

\begin{abstract}
Xavier, D.A.; Barcellos, R.L.; Berrêdo, J.F. \& Silveira, O.M. (2015). Caracterização sedimentar subsuperficial recente da planície costeira amazônica: O exemplo da região do Cabo Norte - AP. Braz. J. Aquat. Sci. Technol. 19(2). eISSN 1983-9057. DOI: 10.14210/bjast.v19n2. The research aims to characterize and to understand the longitudinal and vertical characteristics of cores to interpret the marine or continental influences during the sedimentary processes in estuarine system by mean of grain size analysis and the organic matter contents. Seven cores were collected along the Sucuriju River and sub-sampled observing of the texture differences deposits. The results demonstrated the Sucuriju region presented a progradation of coastline and now the Piratuba Lake is $22 \mathrm{~km}$ in a straight line from the Atlantic Ocean. This register was observed in the core located towards upstream on Sucuriju River that registered a change in the reduction of energy hydrodynamics. Which varies from a prevailing estuarine sedimentation, with high percentage sands and hydrodynamic, to pelitic sedimentation associated to deposition of fine sediments, percentage of organic matter and low intensity hydrodynamics typical of lacustrine environmental. The atmospheric, oceanic and Amazon River forcing are determinant factors in recent sedimentary dynamics and modifications of coastline of the state.
\end{abstract}

Keywords: Amazon River, Estuarine sedimentation, Organic matter, Hydrodynamic.

\section{INTRODUÇÃO}

A planície costeira amapaense está sujeita a várias condições físicas limitantes, submetidas às conjunções das forçantes atmosféricas, oceânicas e amazônicas controladores dos processos oceanográficos físicos e sedimentológicos (Silveira, 1998). A ação dessas forçantes é determinante no funcionamento das estruturações dos espaços litorâneos, apresentando uma grande instabilidade da linha de costa amazônica e, por consequência, ecológica (Allison et al., 1995a; Allison et al., 1996; Allison \& Nittrouer, 1996).

A região costeira do Estado do Amapá está localizada no extremo norte do Brasil fazendo fronteira ao norte com a Guiana Francesa, ao leste pelo Oceano Atlântico e a sul com o rio Amazonas. Estende-se por aproximadamente $750 \mathrm{~km}$ entre as desembocaduras

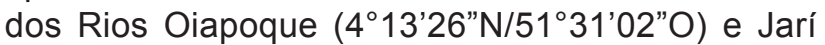
(1 $\left.{ }^{\circ} 9^{\prime} 28^{\prime \prime} S / 51^{\circ} 53^{\prime} 54^{\prime \prime O}\right)$ (Silveira, 1998). Esta região está sob influência sazonal das flutuações de descarga do rio Amazonas e do regime de macromarés semidiurnas apresentando os maiores registros de marés ao longo da zona costeira brasileira com amplitudes variando de 5 a 12 m (Santos, 2006) e correntes costeiras acima de $35 \mathrm{~cm} \cdot \mathrm{s}^{-1}$ (Allison et al., 1996).

O processo de sedimentação nesta planície costeira é reflexo do somatório dessas forçantes atuantes na região. Mendes (1994), Alisson et al. (1995a) e Guimarães et al. (2010) observaram um processo de acresção de um banco lamoso na região de Calçoene, com taxas de sedimentação variando entre $0,33-1,3$ $\mathrm{mm} . \mathrm{ano}^{-1}$ para a planície de Goiabal (Guimarães et al., 2010) e 2 - $4 \mathrm{~cm}^{\text {.ano-1 }}{ }^{-1}$ no cabo do Cassiporé (Kuel et al., 1996). Althammaer et al. (2010) registraram valores de taxa de sedimentação de $0,8 \mathrm{~cm} \cdot$ ano $^{-1}$ no município de Macapá-AP. Allison et al. (1990) identificaram processos de erosão e deposição influenciados pela vazão do rio Amazonas ao longo da planície costeira amapaense.

Por se tratar de um ambiente sob constante influência de forçantes ambientais, a análise granulométrica do sedimento pode ser um indicador do comportamento do agente deposicional e possivelmente relacionar sua intensidade hidrodinâmica, tornando-se essencial no entendimento desse ambiente deposicional (Pettijohn et al., 1987). O diâmetro médio, grau de seleção e assimetria são parâmetros granulométricos que podem fornecer informações sobre esses níveis de energia hidrodinâmica, refletidos 
no predomínio do tamanho do grão, distribuição das partículas e a predominância da fração sedimentada, respectivamente. (Folk \& Ward, 1957; Duane,1964; Suguio, 1980).

Um fator biológico atuante de forma positiva no acúmulo de sedimentos finos é o manguezal. Suas raízes formam estruturas que induzem uma redução no fluxo de água na interface sedimento-água (Furukawa et al., 1997; Marchand et al., 2004; Berrêdo et al., 2008).

O Lago Piratuba é o principal corpo hídrico responsável por exportar água doce para o estuário do rio Sucuriju. Anteriormente, este lago tinha conexão direta com o Oceano Atlântico e, atualmente, encontra-se aproximadamente a $22 \mathrm{~km}$ de distância em linha reta da atual linha de costa. O método de recuperação de testemunhos realizados ao longo do rio é importante para a observação de registros de mudanças no tipo de sedimentação ocorrida nessa região, através da descrição macroscópica, análise granulométrica e da porcentagem de material orgânico. Desta forma, o objetivo deste estudo é interpretar a intensidade hidrodinâmica sedimentar e identificar a influência marinha e/ou fluvial durante os processos sedimentares do estuário do rio Sucuriju Estado do Amapá.

\section{MATERIAL E MÉTODOS}

\section{Características da área de estudo}

A Planície Costeira do Amapá está inserida no limite de três reservas federais: Reserva Biológica do Lago Piratuba (3.570 km²), Parque Nacional do Cabo Orange $\left(6.190 \mathrm{~km}^{2}\right)$ e Estação Ecológica MaracáJipioca $\left(720 \mathrm{~km}^{2}\right)$, gerenciadas pelo Instituto Brasileiro de Meio Ambiente e Recursos Naturais Renováveis (IBAMA) (Silveira, 1998) (Figura 1).

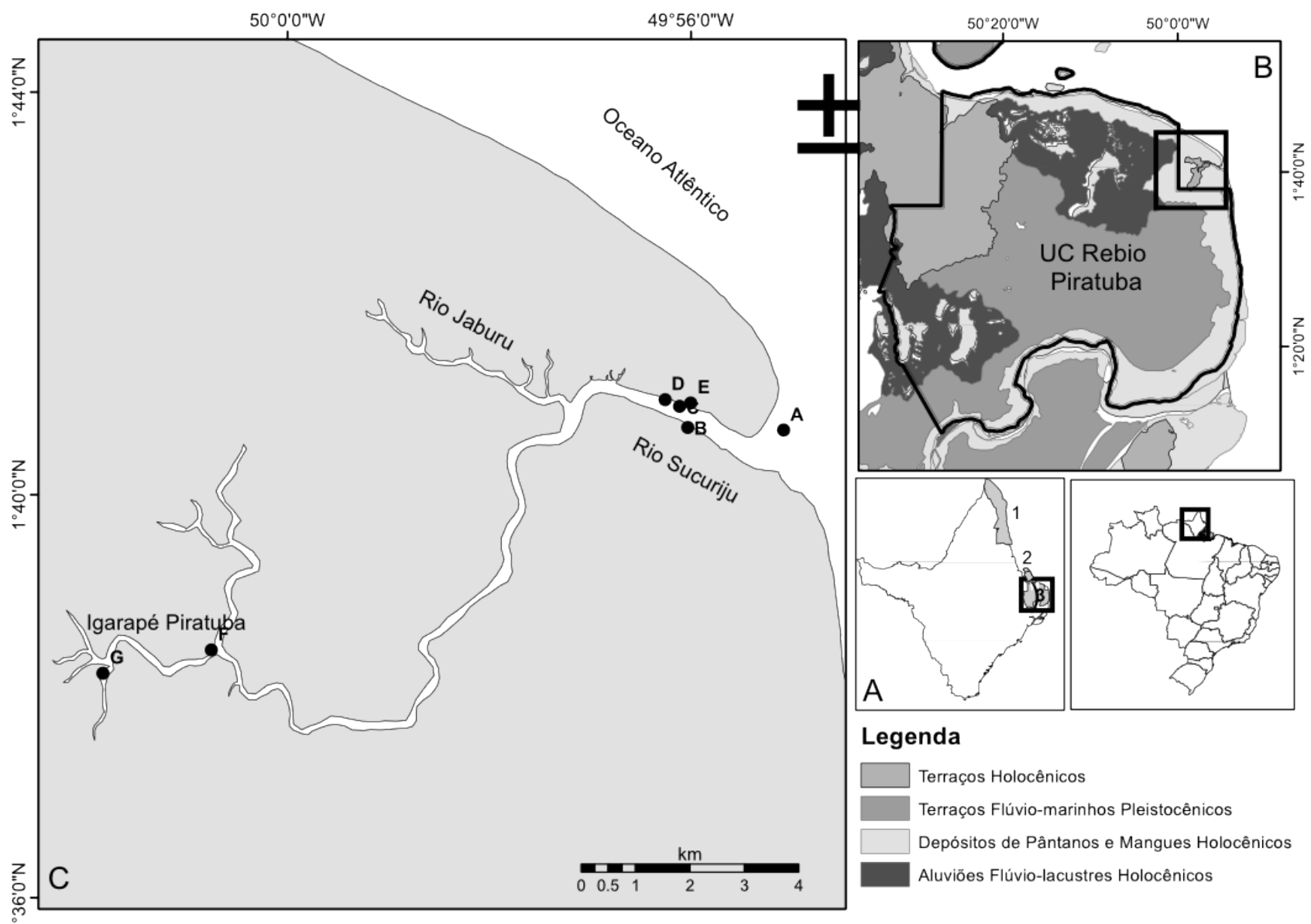

Figura 1 - Localização da área de estudo. (A) 1 - Parque Nacional do Cabo Orange (Calçoene), 2 - Estação Ecológica Maracá-Jipióca e 3 - Reserva Biológica do Lago Piratuba. (B) Limites da REBIO Piratuba e a classificação dos ambientes geológicos. (C) Localização dos testemunhos recuperados ao longo do rio Sucuriju-AP.

O Distrito de Sucuriju está localizado na região do Cabo Norte no extremo leste do Estado do Amapá, entre os paralelos $01^{\circ} 39^{\prime} 49^{\prime \prime} \mathrm{N}$ e $49^{\circ} 55^{\prime} 43^{\prime \prime} \mathrm{W}$. Possui área de 16.700 ha (167 km2), localizada na margem direita do rio Sucuriju, próximo a sua foz, encontra-se $220 \mathrm{~km}$ da capital do estado (Xavier et al., 2012). A 
vegetação é caracterizada por bosques de manguezal, que são influenciados pela topografia e pelos períodos de inundação. Ocorre também a presença de bosques mistos na foz do rio, dominados por Rhizophora sp. e Avicennia sp. (Costa Neto et al., 2007) drenados por canais de maré.

O padrão climático para a região apresenta altos índices térmicos do tipo megatérmico, caracterizado por temperaturas elevadas ao longo do ano com médias oscilando entre $26^{\circ} \mathrm{C}$ a $28^{\circ} \mathrm{C}$. A estação chuvosa é definida por duas estações sazonais, ocorrendo entre os meses de janeiro a julho e a estação seca, de agosto a dezembro, com índice pluviométrico em torno de 3000 mm.ano ${ }^{-1}$ (Peres et al., 1974; Bezerra et al., 1990).

A planície costeira é dominada pelo regime de macromarés que imprimem uma forte hidrodinâmica, além da ocorrência esporádica de pororocas, fenômeno associado à ação da maré, caracterizado pela entrada de ondas no estuário. Ocorre geralmente durante as marés de sizígia, potencializadas nos períodos de equinócios (Chanson, 2005; Santos, 2006), atua sobre a planície costeira. Os processos evolutivos dessa região têm uma relação intrínseca com a evolução geológica e tectônica regional (Allison et al., 1990; Allison et al., 1995a; Costa \& Silveira, 1998).

Duas unidades geológicas foram identificadas por Silveira (1998) para a região do Cabo Norte, o Período Terciário caracterizado pela Formação Barreiras, com sedimentos argilosos a arenoargilosos, com aspecto mosqueado de coloração amarelho-avermelhado a avermelhadas. O Período Quaternário, composto por sedimentos incosolidados quartzosos e lamosos (silte e argila), de coloração cinza claro a cinza escuro.

\section{Amostragem e Análises}

As coletas foram realizadas em dezembro de 2006, utilizando um amostrador pontual por vibração (vibracorer), em pontos pré-definidos desde a foz do rio Sucuriju (A) até a sua porção mais interna (G), totalizando sete testemunhos recuperados, com diferentes profundidades (Tabela 1).

Tabela 1 - Pontos de amostragem, longitude, latitude e profundidade das amostragens de subsuperfície.

\begin{tabular}{c|r|r|r}
\hline Testemunhos & Latitude (N) & Longitude (W) & \multicolumn{1}{|c}{ Prof. (cm) } \\
\hline A & 1,677319 & $-49,917947$ & 368 \\
\hline B & 1,677783 & $-49,933811$ & 426 \\
\hline C & 1,681267 & $-49,935150$ & 140 \\
\hline D & 1,682360 & $-49,937555$ & 126 \\
\hline E & 1,681808 & $-49,933277$ & 81 \\
\hline F & 1,640919 & $-50,012556$ & 330 \\
\hline G & 1,637093 & $-50,030511$ & 230 \\
\hline
\end{tabular}

Em laboratório, os testemunhos foram seccionados longitudinalmente e amostrados obedecendo às diferenças entre as camadas sedimentares observadas pela descrição macroscópica. Em seguida, foram realizados os procedimentos de lavagem para eliminação da salinidade presente no sedimento a fim de evitar interferências no peso e na floculação das partículas finas (silte e argila) (Wentworth, 1922; Suguio, 1980).

Para a obtenção das porcentagens de matéria orgânica obedeceu-se a metodologia proposta por Byers et al. (1978), que consiste na calcinação do material sedimentar em mufla a $500^{\circ} \mathrm{C}$, por quatro horas. As quantificações das porcentagens da fração granulométrica foram realizadas no granulômetro a laser modelo ANALYSETTE 22 MICRO TEC PLUS, com intervalo de leitura de $0,08 \mu \mathrm{m}$ a $2000,00 \mu \mathrm{m}$.
Posteriormente, foram realizadas as análises estatísticas de Folk \& Ward (1957), classificação de Shepard (1954) e Pejrup (1988).

\section{RESULTADOS}

De acordo com a descrição macroscópica, as camadas foram identificadas formando laminações de lama e areia fina, camadas de lama sem estruturas sedimentares (maciças), camadas de areia fina sem estruturas sedimentares (maciça) e camadas de matéria orgânica particulada caracterizadas como turfa (Tabela 2). 
Braz. J. Aquat. Sci. Technol., 2015, 19(2).

Tabela 2 - Descrição dos testemunhos coletados ao longo do rio Sucuriju.

\begin{tabular}{|c|c|c|c|c|c|}
\hline \multirow{16}{*}{ 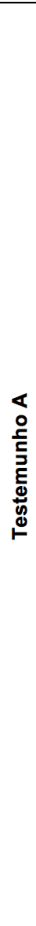 } & Intervalo (cm) & Descrição Macroscópica & \multirow{7}{*}{ 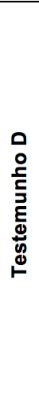 } & Intervalo (cm) & Descrição Macroscópica \\
\hline & $0-35$ & $\begin{array}{l}\text { Laminações de lama e } \\
\text { areia fina com matéria } \\
\text { orgânica }\end{array}$ & & $0-13$ & $\begin{array}{l}\text { Laminações de lama e areia } \\
\text { fina }\end{array}$ \\
\hline & $35-37$ & Turfa & & 13-16 & Silte e argila \\
\hline & $37-48$ & $\begin{array}{l}\text { Laminações de lama e } \\
\text { areia }\end{array}$ & & $16-37$ & $\begin{array}{l}\text { Laminações de lama e areia } \\
\text { fina }\end{array}$ \\
\hline & $48-54$ & Turfa & & $37-53$ & Areia fina \\
\hline & $54-59$ & Silte argila & & $53-65$ & Silte e argila \\
\hline & $59-65$ & Areia fina & & $65-126$ & $\begin{array}{l}\text { Laminações de lama e areia } \\
\text { fina }\end{array}$ \\
\hline & $65-68$ & Turfa com areia fina & & Intervalo & Descrição Macroscópica \\
\hline & $68-70$ & Areia fina & $\Psi$ & $0-6$ & $\begin{array}{l}\text { Laminações de lama e areia } \\
\text { fina }\end{array}$ \\
\hline & $70-72$ & Turfa com areia fina & $\stackrel{\circ}{=}$ & $6-27$ & Silte e argila \\
\hline & $72-74$ & Areia fina & 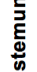 & $27-30$ & $\begin{array}{l}\text { Laminações de lama e areia } \\
\text { fina }\end{array}$ \\
\hline & $74-75$ & Turfa com areia fina & $\stackrel{0}{\vdash}$ & $30-55$ & Silte e argila \\
\hline & $75-79$ & Areia fina & & $55-81$ & $\begin{array}{l}\text { Laminações de lama e areia } \\
\text { fina }\end{array}$ \\
\hline & $79-97$ & Silte e argila & & Intervalo (cm) & Descrição Macroscópica \\
\hline & $97-365$ & $\begin{array}{l}\text { Laminações de lama e } \\
\text { areia fina }\end{array}$ & & $0-70$ & $\begin{array}{l}\text { Silte e argila com matéria } \\
\text { orgânica }\end{array}$ \\
\hline & $365-368$ & Turfa & 눈 & $70-140$ & $\begin{array}{l}\text { Laminações de lama e areia } \\
\text { fina }\end{array}$ \\
\hline$m$ & Intervalo (cm) & Descrição Macroscópica & 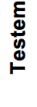 & $140-280$ & $\begin{array}{l}\text { Silte e areia fina com } \\
\text { laminações de } \\
\text { orgânico }\end{array}$ \\
\hline 을 & $0-29$ & $\begin{array}{l}\text { Laminações de lama } \mathrm{e} \\
\text { areia fina }\end{array}$ & & $280-330$ & $\begin{array}{l}\text { Laminações de lama e areia } \\
\text { fina }\end{array}$ \\
\hline 离 & $29-56$ & Areia fina & & Intervalo (cm) & Descrição Macroscópica \\
\hline$\stackrel{\Theta}{\llcorner}$ & $56-395$ & $\begin{array}{l}\text { Laminações de lama e } \\
\text { areia fina }\end{array}$ & 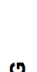 & $\begin{array}{l}0-97 \\
\text { Silte e argila co }\end{array}$ & presença de matéria orgânica \\
\hline & $395-424$ & Silte e argila & $\stackrel{ }{ }$ & e evidencia de & idação \\
\hline & Intervalo $(\mathrm{cm})$ & Descrição Macroscópica & $\overrightarrow{\boldsymbol{\varepsilon}}$ & & \\
\hline 옫 & $0-78$ & $\begin{array}{l}\text { Laminações de lama e } \\
\text { areia fina }\end{array}$ & 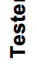 & $\begin{array}{l}97-240 \\
\text { Laminações de }\end{array}$ & ma e areia fina \\
\hline 步 & $78-126$ & Areia fina & & & \\
\hline$\stackrel{0}{\bullet}$ & $126-140$ & Turfa & & & \\
\hline
\end{tabular}

Em todos os testemunhos analisados, o diâmetro médio variou de silte médio a areia muito fina, o grau de seleção variou entre 0,99 a 2,40 (moderadamente selecionado a muito pobremente selecionado, respectivamente). Os valores de assimetria variaram entre simétrico a muito positivo. Os resultados de curtose variaram entre platicúrticos a leptocúrticos. De acordo com a classificação de Shepard (1954) os sedimentos variam entre silte a areia síltica (Tabela 3 ).

Tabela 3 - Análise estatística dos dados granulométricos dos 7 testemunhos recuperados. Diâmetro Médio (D.M), Grau de Seleção (G.S), Assimetria (Assim.), Curtose (Curt.), \% Areia (\%Ar), \% Silte (\%Sil), \% Argila (\%Arg), \% Matéria Orgânica (\%M.O); Shepard: Sar - Silte arenoso, As - Areia síltica, S - Silte.

\begin{tabular}{c|l|l|l|l|l|l|l|l|l}
\hline Test. & D.M & G.S & Assim & Curt & \%Ar & \%Sil & \%Arg & \%M.O & Shepard \\
\hline A & $3,63-5,26$ & $1,52-2,40$ & $0,13-0,27$ & $0,80-1,32$ & $9,49-46,94$ & $39,67-71,55$ & $8,74-13,52$ & $2-9,50$ & Sar-S \\
\hline B & $4,37-5,58$ & $1,10-2,28$ & $0,05-0,25$ & $0,81-1,39$ & $12,76-37,92$ & $44,83-78,60$ & $2,59-11,10$ & $2-9,00$ & Sar-S \\
\hline C & $3,55-5,90$ & $1,31-2,16$ & $0,12-0,26$ & $0,83-1,22$ & $11,66-66,45$ & $31,10-75,20$ & $1,58-14,65$ & $2,50-31,19$ & As - Sar \\
\hline D & $3,65-4,92$ & $0,99-1,99$ & $0,06-0,39$ & $0,97-1,35$ & $33,94-65,65$ & $34,20-57,54$ & $0,15-9,73$ & $3,50-6,00$ & As-Sar \\
\hline E & $4,49-5,45$ & $1,42-2,01$ & $0,18-0,28$ & $0,87-1,16$ & $24,35-44,49$ & $51,44-69,15$ & $4,07-13,80$ & $6,00-9,00$ & Sar \\
\hline F & $4,25-5,18$ & $1,74-2,03$ & $0,20-0,35$ & $0,90-1,16$ & $24,67-39,70$ & $37,47-67,24$ & $6,63-9,03$ & $4,49-7,50$ & As - Sar \\
\hline G & $3,91-5,75$ & $1,34-2,09$ & $0,16-0,33$ & $0,87-1,26$ & $10,89-49,09$ & $41,04-72,33$ & $3,31-13,20$ & $4,00-13,99$ & As-S \\
\hline
\end{tabular}


Dentre os testemunhos analisados, o testemunho $\mathrm{G}$ apresentou variações entre as deposições das camadas mais expressivas durante a sedimentação. No intervalo sedimentar entre $95 \mathrm{~cm}$ a $239 \mathrm{~cm}$, a camada foi descrita com presença de laminação de lama e areia fina, classificada como silte arenoso (Shepard, 1954). Essas estruturas observadas no testemunho $G$ são classificadas como do tipo plano paralelas onde ocorre a deposição de diferentes materiais sedimentares, típicas de ambientes influenciados por maré.

No mesmo testemunho, o intervalo entre $0 \mathrm{~cm}$ a $95 \mathrm{~cm}$ foi descrito como uma camada de silte e argila com estrutura maciça, presença de material orgânico e foi classificado como silte de acordo com Shepard (1954). Neste testemunho foi observada mudança no padrão de sedimentação, transitando antes de um ambiente de maior energia hidrodinâmica influenciado pela maré passando para um ambiente de menor energia, do tipo lagunar, com deposição de sedimentos pelíticos e material orgânico (Figura 2).

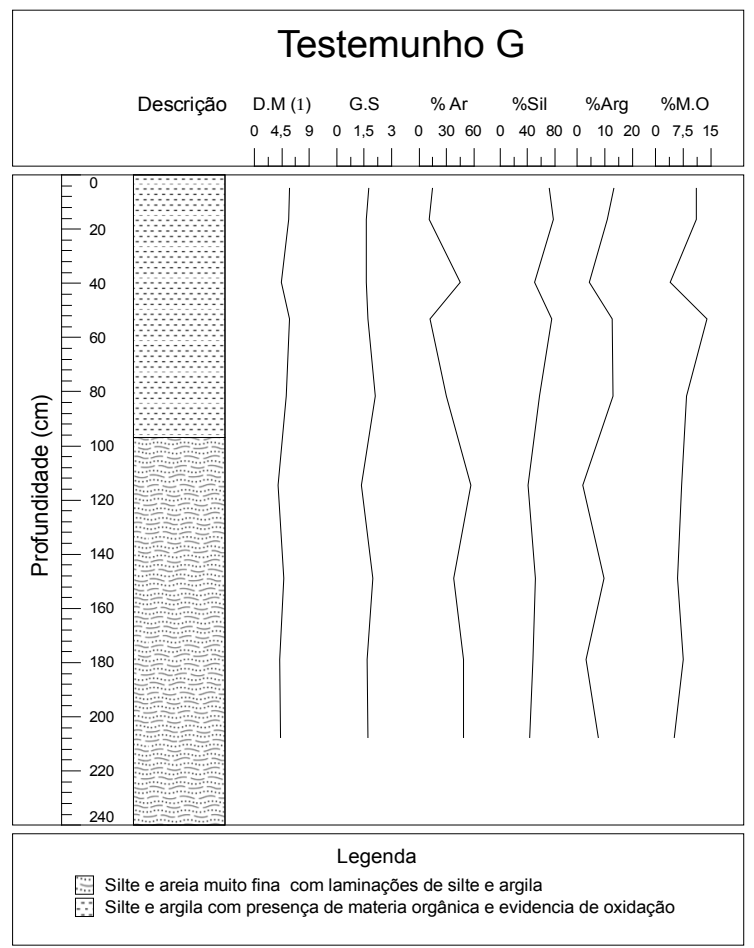

Figura 2 - Descrição visual do testemunho $\mathrm{G}$ e a variação vertical dos parâmetros: diâmetro médio (D.M), grau de seleção, assimetria (Assim.), Curtose, \% das frações (Areia, Silte e Argila) e \% de Matéria Orgânica (\% M.O)

Os testemunhos $\mathrm{A}$ e $\mathrm{D}$, localizados nas proximidades da foz e no médio estuário do rio Sucuriju, respectivamente, estão sob constante influência da oscilação de maré e apresentaram maior variação entre as camadas depositadas, desde camadas com predomínio de areia fina a camadas compostas somente por matéria orgânica (turfa). Quanto à presença de estruturas, variaram de laminações plano-paralelas à maciça. A intensidade hidrodinâmica é um dos fatores responsáveis por essas diferenças entre as camadas sedimentares durante a sedimentação (Figura 3 e 4).

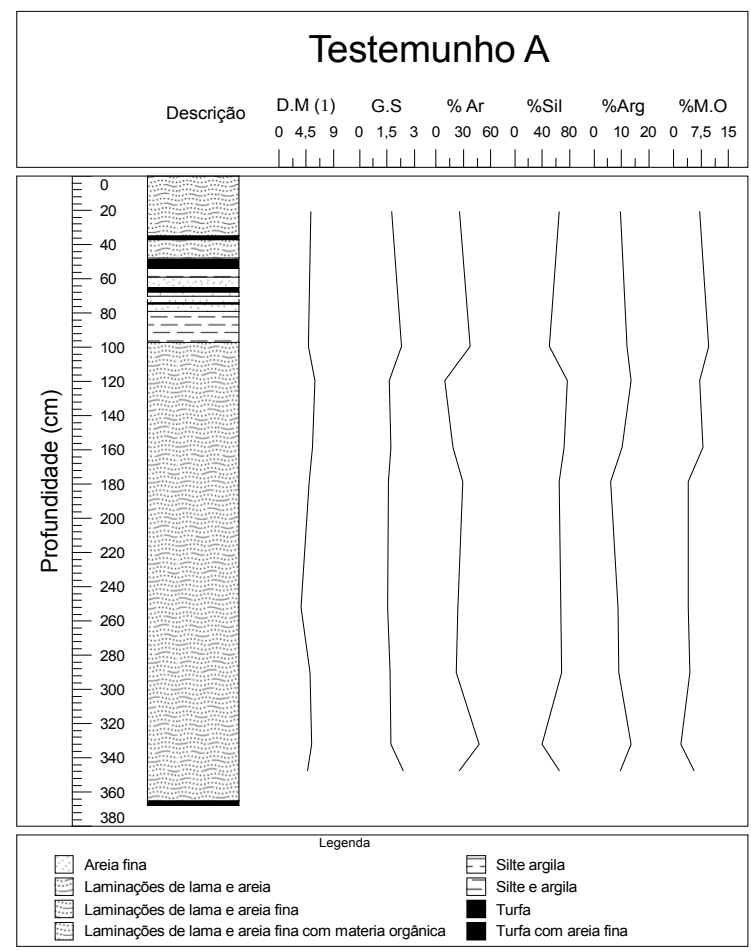

Figura 3 - Descrição visual do testemunho A e a variação vertical dos parâmetros: diâmetro médio (D.M), grau de seleção, assimetria (Assim.), Curtose, \% das frações (Areia, Silte e Argila) e \% de Matéria Orgânica (\% M.O).

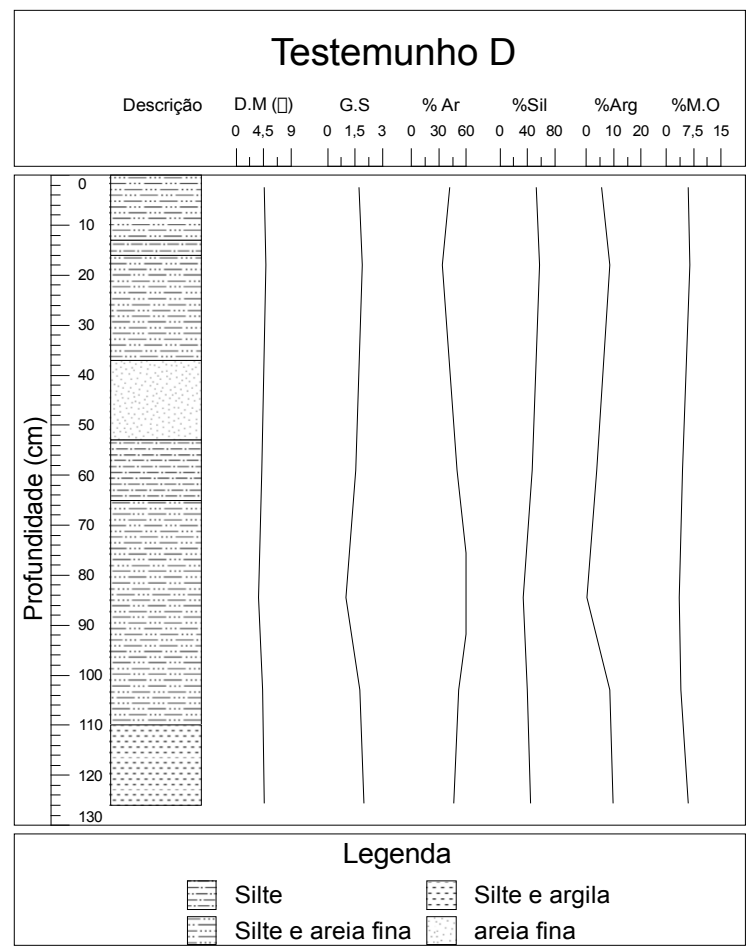

Figura 4 - Descrição visual do testemunho $D$ e a variação vertical dos parâmetros: diâmetro médio (D.M), grau de seleção, assimetria (Assim.), Curtose, \% das frações (Areia, Silte e Argila) e \% de Matéria Orgânica (\% M.O). 


\section{DISCUSSÃO}

Os condicionantes oceanográficos, hidrológicos e amazônicos são os principais agentes na modificação dos parâmetros sedimentológicos e estes também influenciam nas características da linha de costa erosiva ou progradacional (Allison et al., 1995a; Anthony et al., 2010). Em escala geológica, o grande aporte de sedimentos lamosos, oriundos do rio Amazonas, gerou, no Holoceno, estruturas do tipo clinoform e atinge seu desenvolvimento máximo na sua foz, importante na progradação da linha de costa das Guianas à Venezuela (Allison \& Nittrouer, 1998).

Nessa estrutura clinoform, a parte mais elevada é a linha de costa, os processos erosivos trouxeram esses depósitos sedimentares recentes ao nível do mar. No Amapá, esses depósitos erodiram entre 5-10 m e evoluíram para depósitos progradantes, principalmente nas regiões de deltas e na linha de costa do estado (Allison et al., 1995a; Walsh \& Nittrouer, 2009).

De acordo com a descrição dos testemunhos, as estruturas registradas evidenciaram dois tipos de deposição. A primeira, influenciada por uma maior intensidade hidrodinâmica, atuante na parte interna do estuário, representado pelas estruturas laminares. A segunda, influenciada por uma menor energia hidrodinâmica, evidenciada pela deposição de sedimentos finos e material orgânico, com estrutura maciça.

Essas estruturas laminares registradas no estuário do rio Sucuriju foram observadas nas proximidades da foz do rio Amazonas por Kuehl et al. (1986) que registraram camadas intercaladas por fácies lamosas e arenosas, e camadas com finas laminações na região do Cabo Norte. Para Silveira (1998), essas estruturas registradas no estuário são evidências de planícies fluvio-marinha que apresentam características de sedimentos siltíco-arenosos com laminações plano-paralelos e de depósitos lagunares com deposição de sedimento argilo-siltíco e siltíco-argiloso, com altos teores de matéria orgânica.

O diâmetro médio variou verticalmente em todos os testemunhos evidenciando as diferentes intensidades da hidrodinâmica, este associado diretamente ao padrão climático (Silveira, 1998; Santos, 2006). Durante os períodos de inverno amazônico, os elevados índices pluviométricos aumentaram a vazão fluvial na calha do rio. O aumento do volume de água doce dificulta a entrada da maré dinâmica nesse sistema, facilitando assim, o acúmulo de sedimentos finos oriundos dos produtos da lixiviação das margens do rio Sucuriju e do Lago Piratuba.

Nos períodos de estiagem amazônica ocorreu o declínio dos índices pluviométricos e a diminuição da vazão fluvial, fatores favoráveis para o aumento da intensidade da maré dinâmica no interior do estuário.
Desta maneira, esses fatores facilitaram no acréscimo nas porcentagens de areia e na formação das estruturas laminares presentes nas camadas durante a sedimentação, evidenciados nas proximidades da foz do rio Sucuriju (Kuehl et al., 1986; Allison et al., 1995a, Silveira, 1998). Os resultados obtidos nas análises granulométricas foram similares aos obtidas por Kuehl et al. (1995), Allison et al. (1995b), Mendes (1994) e Silveira (1998) em estudos com testemunhos na zona costeira do Amapá.

O rio Amazonas é um dos principais fatores atuantes na configuração da região costeira amapaense (Allison et al., 1996) e determina áreas de acresção (lamosa ou arenosa) e de erosão ao longo da região costeira do estado (Kuehl et al., 1996; Nittrouer et al., 1995). Para a região do Sucuriju essa evolução da linha de costa foi relatada pelos antigos moradores da região, os quais afirmam que existia um contato direto entre o Lago Piratuba e o Oceano Atlântico (Dias, 2002). Esse evento está registrado no testemunho $G$ localizado nas proximidades do Lago Piratuba.

$\mathrm{O}$ registro no testemunho $\mathrm{G}$ demonstra a mudança no tipo de sedimentação, de planície fluviomarinha, com sedimento siltíco arenoso e laminações plano paralelas de lama e areia fina para uma sedimentação típica de depósitos lacustres, com sedimentos siltíco-argilosos e estrutura sedimentar maciça, rica em matéria orgânica. Essas mesmas evidências foram encontradas nos trabalhos de Allison et al. (1996), Kuehl et al. (1996), Guimarães et al. (2010) e Althammer et al. (2010), cujas taxas de sedimentação variaram entre $0,33-1,3 \mathrm{~mm}$ ano $^{-1}$ (Guimarães et al., 2010) a $2-4$ cm.ano-1 (Kuehl et al., 1996), no Cabo Cassiporé (AP).

De acordo com os processos de acresção e progradação da linha de costa relatados para área, são esperadas altas taxas de sedimentação $(>2$ $\mathrm{cm}$.ano ${ }^{-1}$ ) similares aos do Cabo Cassipore (AP), região localizada ao norte da área de estudo. Como não foram obtidos os valores de datação absoluta para os 7 testemunhos estudados, assumiu-se para a área um valor de 2,0 cm.ano-1, a mesma ordem de grandeza observadas por Allison et al. (1996), Kuehl et al. (1996), Guimarães et al. (2010) e Althammer et al. (2010). Desta maneira, pode-se estimar a idade média da base dos testemunhos em torno de 40 anos (testemunho E), à aproximadamente 200 anos (testemunho B), da data de amostragem em campo.

O estuário do rio Sucuriju está submetido à influência de energia hidrodinâmica alta a muito alta. Essa classificação baseia-se nas porcentagens de areia, silte e argila obtidas na classificação granulométrica (Pejrup,1988) que possibilitou a compreensão dos eventos sedimentares ocorridos no estuário do rio Sucuriju. As evidências deposicionais registradas nas diferentes camadas sedimentares indicaram diferen- 
ças na energia hidrodinâmica de sedimentação e um evento de progradação da linha de costa, evidenciada pelos relatos da população local (Dias, 2003).

$\mathrm{O}$ resultado do testemunho $\mathrm{G}$ registrou a mudança da energia hidrodinâmica de sedimentação, confirmado pela variação no gradiente de deposição de sedimento arenosos para sedimentos pelíticos, onde o principal agente de transporte sedimentar em toda a região costeira do Amapá é a forçante amazônica (Kuehl et al., 1995; Allison et al., 1995b; Nittrouer et al., 1995).

A variação na deposição verificada nos testemunhos localizados próximos à foz do estuário é a resposta à entrada da maré dinâmica no sistema. A intensidade da maré é amortecida em direção a montante, ocorrendo diferenças deposicionais ao longo do rio. Desta maneira, a foz representa um ambiente mais dinâmico influenciado pelas marés e ondas, influenciando no aumento das porcentagens da fração areia. A montante, essa intensidade hidrodinâmica é menor o que facilita os acréscimos das porcentagens da fração fina.
Os pontos A, C, D e G apresentaram acréscimos nas porcentagens de areia ao longo dos testemunhos. Essa contribuição siliciclástica é mais evidente nos períodos de estiagem, quando a descarga fluvial é menor e favorece a intrusão da maré dinâmica no interior do estuário, ocasionando o aumento das porcentagens de sedimentos clásticos arenosos (Allisson et al., 1990; Mendes, 1994; Silveira, 1998).

A progradação da linha de costa e a sinuosidade do rio Sucuriju são fatores que colaboraram com o padrão de sedimentação atual imprimindo uma zonação sedimentar no estuário. Assim, os testemunhos $\mathrm{F}$ e G, localizados na porção superior do estuário apresentaram menor intensidade hidrodinâmica e maior influência continental, corroborado por maiores porcentagens de sedimentos da fração fina (silte e argila) e da matéria orgânica. Os testemunhos localizados no baixo estuário ( $\mathrm{A}$ e $\mathrm{B}$ ) e os testemunhos localizados no setor médio do estuário ( $C, D$ e $E$ ) estão submetidos a uma maior intensidade hidrodinâmica e maior influência marinha, comprovado pelos pulsos de areia dentro do sistema (Figura 5).

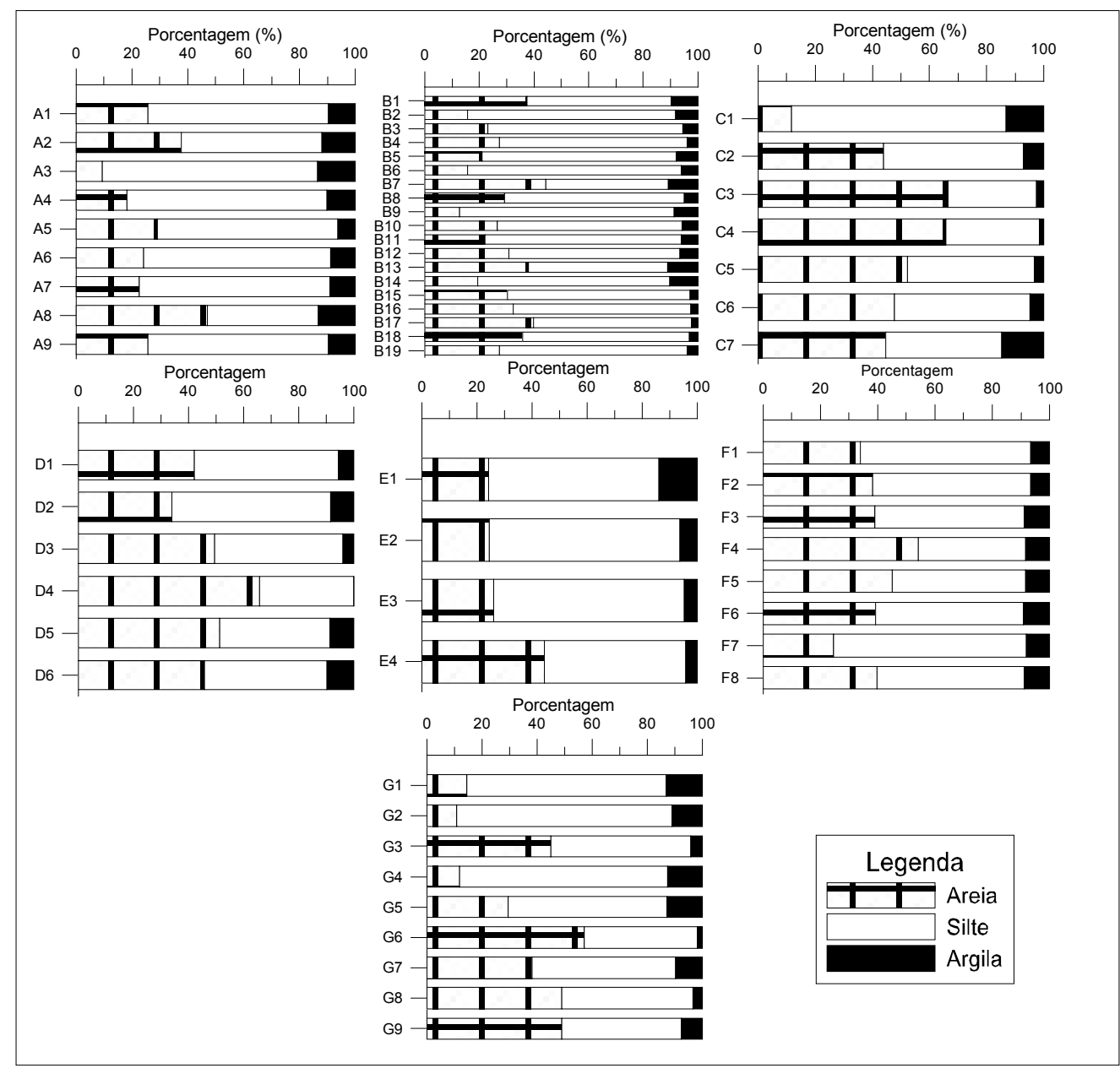

Figura 5: Gráfico de porcentagens de areia (quadriculado), silte (branco) e argila (preto) em relação a profundidade dos testemunhos A, $B, C, D, E, F$ e G. 


\section{CONCLUSÕES}

O estuário do rio Sucuriju apresentou três setores de sedimentação. O primeiro engloba os testemunhos localizados na foz (A e B), influenciados pela forte hidrodinâmica e pela ação direta de condicionantes físicos marinhos. O segundo, localizado no setor médio do estuário, engloba os testemunhos $C$, D e E, apresentou estruturas característica de flutuações de maré, com sedimentação fluvio-estuarina e sedimentos de origem marinha e continental oriundo das margens do estuário e do Lago Piratuba.

O último setor localizado na parte superior do estuário, engloba os testemunhos $\mathrm{F} \mathrm{e} \mathrm{G}$. O testemunho $G$ indicou a diferença no padrão de sedimentação que registrou a passagem da sedimentação com influência da maré para a sedimentação com características lacustres, evidenciado pelo aumento nas porcentagens de sedimentos pelíticos. Essa mudança de energia hidrodinâmica é consequência do processo de progradação da linha de costa que distanciou em aproximadamente $22 \mathrm{~km}$ o Oceano Atlântico do Lago Piratuba.

As altas taxas de sedimentação estimadas para a área (> $\left.2 \mathrm{~cm} \cdot \mathrm{ano}^{-1}\right)$, em conjunto com as forçantes atmosféricas, oceânicas e amazônicas são responsáveis pelas modificações ocorridas de forma drástica e contínua na linha de costa do Estado do Amapá durante a Época do Holoceno recente.

\section{AGRADECIMENTOS}

Ao Conselho Nacional de Desenvolvimento Científico e Tecnológico, a Universidade Federal de Pernambuco - Programa de Pós-Graduação em Oceanografia, a Universidade Federal do Pará e ao Museu Paraense Emílio Goeldi.

\section{REFERÊNCIAS}

Allison, M.A.; Nittrouer, C.A.; Rine, J.M.; Vital, H.; Kuehl, S.A.; Faria Jr, L.E.C.; Dias, G.T.M. \& Figueiredo Jr, A.G. 1990. Depocenters of Amazon River sediment along the Amapá Coast-Brazil. AGU Fall Meeting. San Francisco. 23-31.

Allison, M.A.; Nittrouer, C.A. \& Kineke, G.C. 1995a. Seasonal sediment storage on mudflats adjacent to the Amazon River. Mar. Geol. 125, 303-328.

Allison, M.A.; Nittrouer, C.A. \& Faria Jr., L.E.C. 1995b. Shoreline morphology downdrift of the Amazon River mouth. In: Nittrouer, C.A. \& Kuel, S.A. (ed.), Geological Significance of Sediment Transport and Accumulation on the Amazon Continental Shelf.
Mar. Geol. 125: 373-392.

Allison, M.A.; Nittrouer, C.A.; Farias Jr, L.E.C.; Silveira, O.F.M. \& Mendes, A.C. 1996. Sources and sinks of sediments to the Amazon margin: the Amapa coast. Geo. Mar. Lett. 16: 36-40.

Allison, M.A. \& Nittrouer, C.A. 1998. Identifying accretionary mud shore faces in the geologic record: insights from the modern Amazon dispersal system. In: Schieber, J.; Zimmerle, W. \& Sethi, P.S. (eds.). Mudstones and Shales: Recent Progress in Shale Research, Schweizerbart Publishers, Stuttgart, 147-161.

Althammer, S.; Foßhag, E.; Hoffmann, H.M.; Nery J.R.C. \& Bonotto, D.M. 2010. The use of the $210 \mathrm{~Pb}$ method for preliminary evaluating the sedimentation rate in Amazon River mouth. Ann. Warsaw Univ. of Live Sci. SGGW, Land. Reclam, 42(1) 149-157.

Anthony E.J.; Gardel, A.; Gratiot, N.; Proisy, C.; Allison, M.A.; Dolique, F. \& Fromard, F. 2010. The Amazon influenced muddy coast of SouthAmerica: a review of mud-bank - shoreline interactions. Earth-Sci. Rev. 103: 99-121.

Berrêdo, J.F.; Costa, M.L. \& Progene, M.P.S. 2008. Efeito das variações sazonais do clima tropical úmido sobre as águas e sedimentos de manguezais do estuário do rio Marapanim, costa nordeste do Estado do Pará. Acta Amaz.38(3): 473-482.

Bezerra, P. E. L.; Oliveira, W.; Regis, W.D.E.; Brazão, J.E.M.; Gavinho, J. \& Coutinho, R.C.P. 1990. Amazônia Legal: zoneamento das potencialidades e dos recursos naturais. In: INSTITUTO BRASILEIRO DE GEOGRAFIA E ESTATÍSTICA \& SUDAM. Projeto zoneamento das potencialidades dos recursos naturais da Amazônia Legal: geologia, solos e vegetação. Rio de Janeiro.

Byers, C.; Mills, E.L. \& Stuart, P.L. 1978. A comparison of methods of determining organic carbon in marine sediments, with suggestions for standard methods. Hydrobiologia 58(1): 43-47.

Chanson, H. 2005. Mascaret, Aegir, Pororoca, Tidal Bore. Quid? Où? Quand? Comment? Pourquoi? La Houille Blanche 3: 103-114.

Costa, L.T.R. \& Silveira, O.M.F. 1998. The Araguari estuary: an example of tide Dominated Estuary. An. Acad. Bras.Ciênc. 70 (2) 201-211.

Costa Neto, S.V.; Senna, C.S.F.; Tostes, L.C.L. \& Silva, S.R.M. 2007. Macrófitas aquáticas das regiões dos lagos do Amapá, Brasil. Rev. Bras. Biocienc. 5 (2) 618-620.

Dias, T.A.C., 2003. Gestão participativa: Uma alternativa de ecodesenvolvimento para a Reserva Biológica do Lago Piratuba - AP. 135p. 
Dissertação de Mestrado. Universidade de Brasília. Centro de Desenvolvimento Sustentável. Brasília. Acessado em 21 de março de 2012 < http://repositorio.unb.br/bitstream/10482/7392/1/ ARTIGO_Gest\%C3\%A3oParticipativaReserva. pdf>.

Duane, D.B. 1964. Significance of skewness in recent sediments, Western Pamlico Sound, North Carolina. J. Sediment. Petrol. 34 (4): 864-874.

Folk, R.L. \& Ward, W.C. 1957. Brazos river bar: a study in the significance of grain-size parameters. J. Sediment. Res. 27: 3-26.

Furukawa, K.; Wolansli, E. \& Mueller, H. 1997. Currents and sediment transport in mangrove forest. Estuar. Coast. Shelf Sci. 44: 301-310.

Guimarães, J.T.F.; Cohen, M.C.L.; França, M.C.; Lara, R. \& Behling, H. 2010. Modelo of wetland development of the Amapá coast during the late Holocene. An. Acad. Bras. Ciênc. 82(2): 451-465.

Kuehl, S.A.; Nittrouer, C.A. \& Demaster, D.J. 1986. Distribution of sedimentary structures in the Amazon subaqueous delta. Cont. Shelf Res. 6: (1/2), 311-336.

Kuehl, S.A.; Pacioni, T.D. \& Rine, J.M. 1995. Seabed dynamics of the inner Amazon continental shelf: temporal and spatial variability of surficial strata. Mar. Geol. 125: 283-302.

Kuehl, S.A.; Nittrouer, C.A.; Allison, M.A.; Faria Jr, L.E.C.; Dukat, D.A.; Jaeger, J.M.; Pacioni, T.D.; Figueiredo Jr., A.G. \& Underkoffler, E.C. 1996. Sediments deposition, accumulation and seabed dynamics in an energetic fine-grained coastal environment. Cont. Shelf Res. 16. 283-302.

Marchand, C.; Baltzer, F.; Lallier-Vergès, E. \& Albéric, P. 2004. Pore-water chemistry in mangrove sediments: relationship with species composition and developmental stages (French Guiana). Mar. Geol. 208: 361-381.

Mendes, A.C. 1994. Estudo sedimentológico e estratigráfico de sedimentos holocênicos na Costa do Amapá. 269f. Dissertação (Mestrado em Geologia e Geoquímica) - Programa de PósGraduação em Geologia e Geoquímica, Instituto de Geociências, Universidade Federal do Pará, Belém - PA.

Nittrouer, C.A.; Kuehl, S.A.; Sternberg, R.W.; Figueiredo Jr, A.G. \& Faria Jr, L.E.C. 1995. An introduction to the geological significance of sediment transport and accumulation on the Amazon continental shelf. Mar. Geol. 125 (3/4): 177-192.

Pavê, A. 2003. Assises de la Recherche, plateau des Guyanee. Atelier n.1 -Ecossistèmes Côtiers. (não publicado).

Pejrup, M. 1988. The triangular diagram used for classification of estuarine sediments: a new approach. In: Boer, P.L.; Van Gelder, A. \& Nio, S.D. (Ed). Tide-influenced sedimentary environments and facies. D. Reidel, Dordrecht, p.289-300.

Peres, R. N.; Serruya, M.N. \& Vieira, L.S. 1974. Levantamento exploratório de solos da Folha NA/NB.22 - Macapá. In: BRASIL. Departamento Nacional da Produção Mineral. Projeto RADAM. Folha NA/NB.22 - Macapá: geologia, geomorfologia, solos, vegetação e uso potencial da terra. Rio de Janeiro. (Levantamento de Recursos Naturais, 6).

Pettijohn, F.J.; Potter, P.R. \& Siever, R. 1987. Sand and sandstone. Springer study Edition Series. $2^{\mathrm{a}}$ ed. Springer, 552p.

Santos, V. F. 2006. Ambientes costeiros amazônicos: avaliação de modificações por sensoriamento remoto. 2006. 306p. Tese de Doutorado. Universidade Federal Fluminense - UFF. Niterói - RJ.

Shepard, F.P. 1954. Nomenclature base Don sand-siltclays rations. J. Sediment. Petrol. 24(3): 151-158.

Silveira, O.F.M. 1998. A planície costeira do Amapá dinâmica de ambiente costeiro influenciado por grandes fontes fluviais quaternárias. Tese de Doutorado/Instituto de Geociências, Belém - PA, 230p.

Suguio, K. 1980. Rochas sedimentares: Propriedade, gêneses e importância. São Paulo, Edgard Blücher - USP, 500p.

Walsh, J.P. \& Nittrouer, C.A. 2009. Understanding finegrained river-sediment dispersal on continental margins. Mar. Geol. 263: 34-45.

Wentworth, C.K. 1922. A scale of grade and class terms for clastic sediments. J. Geol. 30: 377-392.

Xavier, D.A.; Guerra, N.C.; Berrêdo, J.F. \& Silveira, O.F.M. 2012. Análise do comportamento físicoquímico da água intersticial do sedimento do rio Sucuriju - Cabo Norte - Amapá. Trop. Oceanogr. 40 (1): 119-132.

Submetido: Novembro/2014

Revisado: Outubro/2015

Aceito: Outubro/2015 